\title{
Regulatory T/B Lymphocytes Involved in the Construction of Immune Mechanism in Non-Small Cell Lung Cancer
}

\author{
Zuo Tie ${ }^{1}$, Li Xinlei ${ }^{1}$, Ren Yang ${ }^{1}$ \\ ${ }^{1}$ Yan’an Hospital Affiliated to Kunming Medical University, Kunming, Yunnan, 650051, China
}

Keywords: regulatory T/B lymphocytes; non-small cell lung cancer; immune mechanism

\begin{abstract}
The incidence of non-small cell lung cancer accounts for $86 \%$ of all lung cancer patients. It is a malignant disease that originates from lung cancer cells. In the past few decades, treatment for non-small cell lung cancer has been achieved great results. However, the current treatment efficiency of non-small cell lung cancer is still far below the expectation. The occurrence of non-small cell lung cancer is closely related to the body's immune function. Regulating T/B lymphocyte is an immune system. To regulate the function of cell subsets, this paper combines the study of regulatory T/B lymphocytes in the construction of non-small cell lung cancer immune mechanisms.
\end{abstract}

\section{Introduction}

From a worldwide perspective, since the beginning of the last century, lung cancer has become a more frequent outbreak of cancer diseases, and the incidence and mortality of lung cancer patients in various countries are constantly rising. According to the statistical results of authoritative statistics, there were approximately 14.6 million cancer cases in the world in 2012, of which approximately 1.64 million were lung cancer cases. Moreover, in recent years, the number of new cancer cases in China has become the world's first, accounting for about $21 \%$ of global cancer cases, and the mortality rate accounts for about $26 \%$ of the world's deaths, of which the mortality rate of lung cancer disease is the highest. At present, the incidence and mortality rates of lung cancer in China are on the rise, and are higher than the global average. Lung cancer disease has become the first disease of cancer-related death causes in China in 2008, according to the authoritative data of the National Cancer Research Office. It shows that in recent years there are approximately 560,000 cases of lung cancer in China each year. The relevant report of the World Health Organization studies the reality and predicts that by 2025, the number of people who die from lung cancer disease in China will reach 1 million each year. China will become the world's largest lung cancer country. Of these, Non-small-cell carcinoma cases are usually in stage IIIB or IV at the time of treatment. The chance of radical cure is very small at this stage, and only palliative treatments can be used to relieve the patient's pain, to extend the survival of patients [1]. Numerous clinical studies have demonstrated that regulatory T/B lymphocytes have an irreplaceable role in the development and progression of tumors and have a positive effect on the composition of the immune mechanisms that promote tumors, and have a high research significance.

\section{Regulatory T/B Lymphocytes}

\subsection{Regulatory $\mathrm{T}$ lymphocytes}

Regulatory $\mathrm{T}$ lymphocytes (nature regulatory $\mathrm{T}$ cells), abbreviated as Treg, play an active role in the negative regulation of clinical immune responses and the body's own immune tolerance. Treg is mainly derived from the bone marrow pluripotent stem cell region. Some stem cells in the bone marrow of the body migrate to the thymus-dependent region, and then gradually differentiate and mature under the induction of thymus hormone, thereby growing into $\mathrm{T}$ cells with obvious immunoreactivity. The differentiated and mature $\mathrm{T}$ cells are distributed in the body through the blood flow to the thymus-dependent region. The recycling of the cells is mainly carried out by the 
body's lymphatic vessels, peripheral blood, and organ tissue fluids, thereby stimulating the immune regulatory function of T cells. Treg, on the other hand, suppresses the cellular immune response of the body to maintain the immune tolerance of peripheral organs, so as to suppress the occurrence of autoimmune diseases and limit the development of diseases such as chronic inflammation. However, in the microenvironment of tumor development and development, Treg can play a role by inhibiting effective anti-tumor immunity, thereby promoting the occurrence and development of tumors. Clinical trials in recent years have shown that in non-small cell lung cancer clinical cases, the proportion of Treg in total T lymphocytes in the tumor tissue, peripheral blood, and tissue fluid shows a significant upward trend, which shows that the Treg cells Accumulated in the course of clinical treatment of patients with non-small cell lung cancer, has a close relationship with the development of the tumor.

\subsection{Regulatory B lymphocytes}

Regulatory B lymphocytes (natural regulatory B cells, abbreviated Breg), such cells are mainly derived from the hematopoietic stem cells in the bone marrow of the body, and differentiated and developed on the basis of this. Compared with Treg, Breg is larger in size. Breg is obviously stimulated by antigen, and then it will proliferate and differentiate into a larger number of plasma cells, while plasma cells can synthesize and secrete antibodies and achieve recycling in the blood [2]. Breg accumulated more bone marrow and lymph nodes in the body than Treg, while the number of assembled cells in the peripheral blood was less than that of Treg cells, and there were fewer around the thymus, and only a few Breg involved in cell recycling. In addition, there are many different markers on the cell membrane of Breg, including mainly surface antigens and receptors on the surface, and these superficial dominant markers can be said to be megalin molecules bound to the cell membrane. Regulatory B lymphocytes are newly discovered, definitive regulatory lymphocytes that play an important role in immune regulation at the level of innate and adaptive immunity in the body. Related clinical studies have shown that Breg can inhibit pathological T-lymphocytes through their contact with cells in the development and progression of diseases such as inflammatory bowel disease, rheumatoid arthritis, and cerebrospinal meningitis. Immunoregulatory cytokines such as B lymphocytes act to suppress their harmful immune responses. The immune mechanism of Breg can not only achieve the progress of autoimmune diseases, but also play an important role in inhibiting the anti-tumor immunity in various tumor experimental models. However, so far, the occurrence of Breg in lung cancer diseases such as non-small cell lung cancer Little is known about the research and understanding of the functions and roles in the development process.

\section{Regulatory T / B Lymphocytes and Non-small Cell Lung Cancer}

According to the study of clinical efficacy of regulatory $T$ lymphocytes and non-small cell lung cancer and other cancer diseases in recent years by many scholars and researchers, the number of $\mathrm{T}$ lymphocytes in peripheral blood, tumors, and lymph nodes of these cancer patients was found. There is a clear abnormal increase and there is a negative correlation with the development of cancer patients. Thus, if the clinical function of suppressing the cell function of Treg can be achieved, the recovery of tumor immune function may be achieved. Treg can affect the interaction between tumors and the immune system in cancer cases. Non-small cell lung cancers generally have different degrees of immunosuppression. According to previous experimental studies, tumor cells can induce the proliferation and differentiation of Treg by releasing some factors, and the number of tumors increases with the increase of tumors, and promotes further development of tumors. The regulation of B lymphocytes in various clinical experiments and studies proves that it has obvious immunological negative effects in autoimmune diseases such as inflammatory bowel disease, rheumatoid arthritis disease, and immune cerebrospinal meningitis. Regulatory effects can effectively curb the development of the disease and promote the reversion of the disease, with a clear regulatory role [3]. To regulate the relationship between B lymphocytes and non-small cell lung cancer, according to related studies, Breg can down-regulate the anti-tumor effect of cytotoxic 
Treg, Breg's anti-tumor effect can enhance the body's anti-tumor immunity.

With regard to the relationship between regulatory $\mathrm{T}$ lymphocytes and regulatory B lymphocytes, relevant clinical experiments and studies have shown that Breg can promote the proliferation of Treg, and thus play a role in inhibiting the development of autoimmune. Among them, B7 molecules expressed in regulatory B lymphocyte molecules play an important role in recruiting regulatory $\mathrm{T}$ lymphocytes and then entering the body's central nervous system. It can be seen that the relationship between regulatory $\mathrm{T}$ lymphocytes and regulatory $\mathrm{B}$ lymphocytes is not isolated from each other. They have previously been clearly associated with each other, and promote the close relationship, and this relationship for the function of cells with its irreplaceable role, regulatory T/B lymphocytes have important research significance for the composition of immune mechanisms involved in non-small cell lung cancer.

\section{Regulation of $T$ / B Lymphocytes Involved in Non-small Cell Lung Cancer Immune Mechanism}

Clinical display incidence of cancer in general is a complex disease process involved in the development of a multi-factor and multi-stage lesions and multiple genes, non-small cell lung cancer is no exception. The early symptoms of non-small cell lung cancer include chest pain, blood stasis, low fever, and cough. The late symptoms include mental fatigue, weight loss, and even breathing difficulties, cough, and hemoptysis. Current treatments for non-small cell lung cancer is usually by means of radiation, chemical and surgical means and other means, but the treatment effect is not clinically significant, clinically high mortality rate. In recent years, a large number of experimental studies have shown that the immune circulatory system unexpectedly plays an opposite role in the occurrence and development of tumors and, to a certain extent, promotes the growth and development of tumors. Regulatory $\mathrm{T}$ lymphocytes and regulatory B lymphocytes play a key role in the negative regulation of the immune system response. It can promote tumorigenesis by inhibiting effective antitumor immune responses in the clinical tumor microenvironment. And development.

The study on the regulatory mechanism of regulatory T/B lymphocytes involved in the immunological mechanism of non-small cell lung cancer tumors mainly includes three aspects: the clinical study of regulatory T/B lymphocytes in peripheral blood of patients with advanced non-small cell lung cancer, tumors Microenvironmental studies on the regulation of regulatory T/B lymphocytes in vitro and the regulatory mechanisms of tumour microenvironmental regulation of regulatory T/B lymphocytes. For the clinical study of immune status of non-small cell lung cancer patients, we first explored the relevant clinical studies of peripheral T-cell lymphocytes in patients with advanced non-small cell lung cancer, the results confirmed in the peripheral blood of patients with advanced non-small cell lung cancer. The proportion of naive regulatory $\mathrm{T}$ lymphocytes showed a downward trend, and mature regulatory $\mathrm{T}$ lymphocytes concentrated from peripheral blood to local areas of tumor tissue, thereby promoting the growth of local tumors of non-small cell lung cancer, and further leading to the patient's tumor The load has increased to some extent. In addition, clinical trials have found a significant increase in the proportion of Breg populations, and Breg may inhibit or downregulate protective cytotoxicity through its secreted IL-10, TGF-B, and other cytokines, and through regulation T-lymphocyte response to achieve anti-tumor immune response in the tumor microenvironment [4]. It can be seen that the negative immunoregulation and the co-inhibition of the regulatory T/B lymphocytes involved in the effective anti-tumor immune response can effectively promote the progression of the tumor.

Secondly, in order to investigate the possible role of tumor microenvironment in tumor immunity and the role of inflammation in antitumor immunity, in vitro studies on the regulation of regulatory T/B lymphocytes by the tumor microenvironment were carried out. An in vitro model of co-culture of lung cancer cells activated by clinical inflammation with peripheral blood mononuclear cells, the results confirmed that regulatory $\mathrm{T}$ lymphocytes and regulatory $\mathrm{B}$ lymphocytes and their secreted cytokines in the tumor microenvironment, to achieve its regulation Function and effects, while regulatory T/B lymphocytes play a crucial role in the regulation of inflammatory activated tumor 
cells, and it has a significant correlation with the severity of inflammation. It can be seen that there may be a dynamic network between the tumor microenvironment and the tumor immune response in most clinical settings. The relationship among them is mutual regulation and interaction, and the extent of its effects determines the occurrence and development of tumors. Finally, to investigate the role of regulatory T/B lymphocytes in the immune mechanism of NSCLC tumors, tumor microenvironmental regulation of regulatory T/B lymphocytes was studied. Combined with relevant research results, we confirmed the correlation between cell-derived chemokines, such as cell-expressed and secreted factors, MIP-1a, and regulatory T/B lymphocytes, and their expression levels in lung cancer patients such as non-small cell lung cancer. The prognostic level has a great correlation [5]. The correlation between tumor cell chemokines and regulatory T/B lymphocyte negative immune responses and clinically effective control of tumor-associated inflammatory responses may lead to enhanced tumor immune function, which can improve the tumor to a certain extent. The level of prognosis.

\section{Conclusion}

In summary, with regard to regulatory T/B lymphocytes participating in the study of the immunological mechanism of NSCLC tumors, although some achievements have been achieved, due to time, funding, and technical limitations, our current research has only made the initial stage of exploration still has further in-depth research and improvement in breadth and depth. I hope to further explore the functional role of regulatory T/B lymphocytes in the immune mechanisms of non-small cell lung cancer and other tumor diseases in the course of future follow-up medical research so as to provide new clinical treatment ideas for immunotherapy of tumor diseases. In order to improve the clinical inhibition of tumor diseases.

\section{References}

[1] Lai Fei, Zhang Jing, Lai Dong. Expression and significance of lymphocyte subsets, interleukin-6 and tumor necrosis factor-alpha in peripheral blood of elderly patients with lung cancer[J]. Chinese Journal of Gerontology, 2017, 37(23):5856-5857.

[2] Zhang Yaming relationship regulatory T cells and NK cells in the pathogenesis of preeclampsia variation [J] Foreign Medical Research, 2017,15 (34): 1-2.

[3] Wu Jiawen, Xiao Shengxiang, Ren Jianwen, Huo Jia, An Jingang, Zou Sixin, Zhou Jing. The role of keratin 17 in $\mathrm{T}$ lymphocyte-mediated immune system of psoriasis vulgaris[J]. Chinese Medical Frontiers Magazine (Electronic Edition), 2016, 8(10): 33-36.

[4] He Junnan, Zhao Shousong regulatory $\mathrm{T}$ cells and the relationship between chronic HBV infection [J] Journal of Clinical Hepatology, 2016,32 (02): 361-365.

[5] Li Zongji, Zhao Wei. Differential proteomic analysis of T lymphocytes in mice immunized with 14-3-3 recombinant vaccine of Echinococcus granulosus[J]. International Journal of Laboratory Medicine, 2015,36(23):3388-3389 +3392. 